To appear in Atmosphere-Ocean

Vol. 00, No. 00, Month 20XX, 1-32

\title{
The fluxes and behaviour of plumes inferred from measurements of coherent structures within images of the bulk flow
}

\author{
H. C. Burridge*, J. L. Partridge and P. F. Linden \\ Department of Applied Mathematics and Theoretical Physics, University of Cambridge, Centre for \\ Mathematical Sciences, Wilberforce Road, Cambridge CB3 0WA, UK. \\ (Original manuscript received 00 Month 20XX; accepted 00 Month 20XX)
}

This paper describes how measurements of the movement of identifiable features at the edge of a turbulent plume can be interpreted to determine the properties of the mean flow and consequently, using plume theory, can be used to make estimates of the fluxes of volume (mass), momentum and buoyancy in a plume. This means that video recordings of smoke rising from a chimney or buoyant material from a source on the sea bed can be used to make accurate estimates of the source conditions for the plume. At best we can estimate the volume flux and buoyancy flux to within about 5\% and $15 \%$ of the actual values, respectively. Although this is restricted to the case of a plume rising in a stationary and unstratified environment, we show that the results may be of practical use in other more complex situations. In addition, we demonstrate that large scale (turbulent) coherent structures at the plume edge form on a scale approximately $40 \%$ of the local (mean) plume half-width and travel at almost $60 \%$ of the average local (mean) velocity in the plume.

KEYwords: Convection, Pollution, Physics, Dynamics, Tropopause, Eddies, Dynamics, Plumes in the atmosphere and ocean.

*Corresponding author's email: hcb39@cam.ac.uk 


\section{Introduction}

Plumes or turbulent buoyant jets arise frequently (e.g. Woods, 2010) and their effects are often dramatic and far-reaching, for example the eruption of the Icelandic volcano Eyjafjallajökull in 2010 or the Deepwater Horizon oil leak in the Gulf of Mexico in the same year. Despite the global economic and environmental impact of such events, making accurate predictions of how the flow will evolve continues to prove challenging. We seek to contribute towards improved future predictions by examining a variety of plumes and buoyant jets evolving in a quiescent environment of uniform density. Our experimental measurements, solely, involve movie images of the bulk plume flow, measurements which could be repeated in the field. Our analysis provides new physical insights into an already well studied flow. In the absence of any knowledge of the source conditions or its location, our measurements of the plume width and the velocity at the plume edge enable robust predictions for the fluxes of momentum and volume - the volume (mass) flux being, for example, crucial in assessing the scale of an oil leak. Moreover, examining the spatial derivatives of the momentum flux provides estimates of the source buoyancy flux (see $\S 1 . a)$, which is the critical quantity determining the evolution of the flow.

A plume forms when buoyant fluid is released from a localised source. Less dense plume fluid, of characteristic local density $\rho(z)$ (a function of height $z$ ), rises and entrains fluid from the environment. The relative magnitudes of inertia and buoyancy at the plume source play a key role in determining the dynamics of the flow that evolves. For plumes in the atmosphere or oceans the role of stratification within the environment on the dynamics of a plume is always significant providing the region of interest is of sufficiently large height. Indeed, the effects of the Earth's rotation may affect the dynamics but only at very large scales, typically for horizontal extents of approximately $10-200 \mathrm{~km}$ in the ocean and $1000 \mathrm{~km}$ in the atmosphere. Moreover, the behaviour of these plumes frequently requires consideration of the momentum within the environment to be considered in order to make accurate predictions of the evolution of the plume (Woodhouse, Hogg, Phillips \& Sparks, 2013). Despite these considerations, the understanding 
of plumes in the atmosphere or oceans has greatly benefited from examination of plumes formed under certain idealised conditions.

In this paper we examine one such case, namely, a Boussinesq plume within a quiescent environment of uniform density $\rho_{a}$. Such plumes rise indefinitely (in an unbounded domain) and, irrespective of the conditions at the source, eventually attain an invariant balance between the inertia and buoyancy within the plume, after which the plume is described as being 'pure'. We vary the relative scale of inertia and buoyancy at the source of our plumes and define this ratio by a scaled Richardson number, the plume parameter $\Gamma_{0}$, with $\Gamma_{0}=1$ describing a pure plume source.

When plumes occur naturally or accidentally, or are indeed deliberately employed by industry, theoretical and numerical models are often utilised to make calculations regarding the evolution of the plume and hence predict and control its impact. However, such models require information regarding the conditions of the flow at some height on which to base model calculations. Given the difficulty in predicting natural or accidental releases of buoyant fluid and the propensity for plumes to occur within, or create, inhospitable environments, precise measurements of the detailed flow characteristics within the plume are often difficult to obtain. However, movie imaging of the bulk flow, frequently made visible by the inclusion of particulates or chemicals acting as a tracer within the plume, is often possible in the field at moderate resolutions and frame rates. We restrict our experimental measurements to images of the bulk plume taken at resolutions and frame rates which can be easily replicated in the field, hence enabling our analysis to be deployed on plumes of real consequence.

Theoretical models of self-similar plumes require the radial distribution of velocity and buoyancy to be parameterised. Early studies (e.g. Morton, Taylor \& Turner, 1956) tended to favour the representation of the mean profiles as 'top-hat' profiles with a uniform velocity and buoyancy within the plume and zero outside. More recent experimental studies of plumes (Ezzamel, Salizzoni \& Hunt, 2015; Shabbir \& George, 1994; Wang \& Law, 2002) examined the distributions of the time-averaged velocities $w(r, z)$ and buoyancies $g^{\prime}(r, z)=g\left(\rho_{a}-\rho(r, z)\right) / \rho_{a}$ at points fixed 
in space, where $r$ and $z$ denote the radial and vertical coordinate, respectively. The radial distributions of $w(r, z)$ and $g^{\prime}(r, z)$ are observed to be typically Gaussian-like and this has influenced the choice in more recent models (Craske \& van Reeuwijk, Under consideration). Generally, the choice of distribution for a given theoretical model is of little consequence beyond altering numerical constants. However, insight into the physical process of turbulent entrainment might be improved by better understanding of the dynamics at the plume edge. Time-averaged statistics of measurements at fixed points are inherently influenced by spatial intermittency as the meandering plume turbulently fluctuates in width and temporal intermittency as turbulent structures pass through the region of interrogation. The role of intermittency in generating Gaussian-like distributions for the velocity and buoyancy is unclear (Papantoniou \& List, 1989). For our experiments on turbulent saline plumes stained with dye, mass transport by advection dominates diffusion, i.e. the flow has high Péclet number, and the edge of the plume remains sharp with insignificant effects from diffusion (following Papantoniou \& List, 1989, the Batchelor scale being between $2 \times 10^{-5} \mathrm{~cm}$ and $6 \times 10^{-4} \mathrm{~cm}$ for our experiments). By tracking structures at the plume edge our analysis avoids the need to time-average, thereby mitigating the effects of intermittency and providing information regarding the physical distribution of velocities.

The tracking of coherent structures is of interest within numerous different flows at a wide range of scales (Haller, 2015; Hussain, 1983). However, the process of identifying and tracking such structures can be challenging. Turbulent free shear flows which are significantly constrained in one dimension, either physically or by the effects of stratification, are observed to form large scale coherent structures. For example, quasi-two-dimensional jets have been shown to exhibit an inverse cascade (classically associated with quasi-two-dimensional turbulence) and the coherent structures (eddies) that grow linearly with the distance along the jet axis visibly dominate the flow (Landel, Caulfield \& Woods, 2012). Likewise in quasi-two-dimensional plumes the successful tracking of large scale coherent structures has yielded physical insights into the dynamics (Rocco $\&$ Woods, 2015). However, many of the plumes that develop naturally are better described, for at least a significant portion of their vertical travel, by the dynamics of axisymmetric plumes. For 
such three-dimensional flows a forward cascade (taking energy to the small scales) is expected and so identifying and tracking coherent structures may be inherently more difficult. Some insight has been provided by coherent structure tracking in the mixing layer of axisymmetric pure jets (e.g. Hussain \& Clark, 1981). Furthermore, Papantoniou \& List (1989) investigated the structure of buoyant jets using planar laser-induced fluorescence and provided some interesting insights into the role of intermittency on the scalar concentrations. Their analysis also included a correlation analysis which yielded some limited data for centreline velocities (see their figure 25). Some correlation analysis of images of the bulk flow for plumes and buoyant jets has been carried out and a technique, described as 'optical plume velocimetry', which attempts to track coherent structures within the whole image, has been developed by Crone, McDuff \& Wilcock (2008). This has provided estimates of the plume volume flux which have been of practical use, for example in the case of the Deepwater Horizon oil leak (Crone \& Tolstoy, 2010). However, the optical plume velocimetry data from Crone et al. (2008) did present some bias in the predictions of volume flux and did not provide estimates of the buoyancy flux. We present data for axisymmetric buoyant jets and plumes based on a correlation analysis of images of the bulk flow producing accurate estimates of the fluxes of volume (mass), momentum and buoyancy within the flow. Furthermore, the analysis provides data for the evolution of coherent structures and yields insights on the conditions within the flow local to the plume edge.

The remainder of this paper is laid out as follows. We present the theoretical scalings for the flow in $\S 1$ and provide an overview of the experiments and analysis in $\S 2$ (full details of which are presented in the appendices). The results are presented in $\S 3$ before the implications of our findings discussed and conclusions drawn $(\S 4)$.

\section{1.a Dimensional considerations}

The dynamics of turbulent plumes are determined by the physical fluxes of mass, momentum and the force arising from the buoyancy. The conservation equations for high Péclet number, incompressible (low-Mach number) plumes which differ in density only marginally from the 
ambient $\left(\rho_{a}-\rho \ll \rho_{a}\right)$, i.e. Boussinesq plumes, can be written in terms of the fluxes of volume $Q$, specific momentum $M$ (hereinafter momentum) and buoyancy $F$, defined as

$$
Q=2 \pi \int_{0}^{\infty} r w(r, z) \mathrm{d} r, \quad M=2 \pi \int_{0}^{\infty} r w(r, z)^{2} \mathrm{~d} r, \quad F=2 \pi \int_{0}^{\infty} r w(r, z) g^{\prime}(r, z) \mathrm{d} r
$$

For self-similar plumes in uniform environments, neglecting turbulent transport terms and assuming that the characteristic horizontal velocities of entrained fluid are related to the local characteristic vertical velocities in the plume by a constant, $\alpha$, the conservation equations can be written

$$
\frac{\mathrm{d} Q}{\mathrm{~d} z}=2 \pi^{1 / 2} \alpha M^{1 / 2} \propto 2 \alpha b w, \quad \frac{\mathrm{d} M}{\mathrm{~d} z}=\frac{Q F}{M} \propto b^{2} g^{\prime}, \quad \frac{\mathrm{d} F}{\mathrm{~d} z}=0,
$$

where $b, w$ and $g^{\prime}$ are characteristic half-widths, velocities and buoyancies, respectively, within the plume. Solutions of (2) yield a Richardson number which is invariant for a pure plume and is herein referred to as the plume parameter, defined as

$$
\Gamma=\frac{5}{8 \sqrt{2} \alpha_{G}} \frac{Q^{2} F}{M^{5 / 2}}=\frac{5}{8 \alpha_{T}} \frac{Q^{2} F}{M^{5 / 2}}
$$

where $\alpha_{G}$ and $\alpha_{T}$ denote the entrainment coefficient upon assuming Gaussian and top-hat profiles, respectively, for the radial profiles of the velocity and buoyancy. For completeness, $\sqrt{2} \alpha_{G} \equiv \alpha_{T}$

Morton et al. (1956) made the solution for a point source emanating a steady flux of buoyancy $F_{0}$ (a point source pure plume) in a uniform environment well-known. For such a case there are no obvious independent length and time scales within the flow. However, the vertical distance between the physical source of any pure plume and the 'virtual origin' of a point source plume of matching buoyancy flux scales linearly with the radial scale $Q_{0} / M_{0}^{1 / 2}$, herein the subscript ' 0 ' denotes the conditions at a physical plume source. Hence the natural length scale on which to scale a pure plume is

$$
L_{Q}=\frac{Q_{0}}{M_{0}^{1 / 2}},
$$


and the natural velocity scale is $\left(F_{0} / L_{Q}\right)^{1 / 3}$. Consideration of forced plumes, i.e those dominated by the fluxes of buoyancy $F_{0}$ and momentum $M_{0}$, identifies the jet-length

$$
L_{M}=\frac{M_{0}^{3 / 4}}{F_{0}^{1 / 2}}=\sqrt{\frac{8 \alpha_{T}}{5}} \frac{L_{Q}}{\Gamma_{0}^{1 / 2}},
$$

as the natural length scale and $F_{0}^{1 / 2} / M_{0}^{1 / 4}=\left(F_{0} / L_{M}\right)^{1 / 3}$ as the natural velocity scale. We utilise these scales in the presentation of our data throughout $\S 3$.

Solutions to the conservation equations (2) are typically presented in terms of characteristic scales for the local plume half-width, vertical velocity and buoyancy, for which it is necessary to assume a particular statistical description for the radial distribution of velocity and buoyancy within the plume. Given that we present measurements of velocities at the experimentally determined plume edge we choose to adopt the top-hat description of a plume; the other reasonable choice being that the velocity and buoyancy follow Gaussian distributions, for which (contrary to our experiments) there is no well defined plume edge. On the assumption of top-hat distributions the proportionality signs within the conservation equations (2) can be replaced with equal signs with the inclusion of a factor of $\pi$ on the right hand side - complete solutions of which are presented by Morton et al. (1956).

Throughout, the subscripts ' $G$ ' or ' $T$ ' denote (time-averaged) characteristic scales appropriate assuming the radial profiles of the velocity and buoyancy are of equal width and follow Gaussian or top-hat profiles, respectively. For completeness, the Gaussian centreline velocities and buoyancies are $w_{G} \equiv 2 w_{T}$ and $g_{G}^{\prime} \equiv 2 g_{T}^{\prime}$, respectively, and the plume half-width (at which the velocity has decayed to $\left.w_{G} / e\right), b_{G} \equiv b_{T} / \sqrt{2}$.

\section{Experiments and diagnostics}

The experimental set-up was deliberately simple. Plumes were created by steadily ejecting saline solution containing a coloured dye downwards within a glass sided visualisation tank, of crosssection $80 \mathrm{~cm} \times 100 \mathrm{~cm}$, filled to a depth of $90 \mathrm{~cm}$ with fresh water. Source volume fluxes were 
Source plume parameter

Relatively pure

Moderately Forced

Forced

$$
1 \times 10^{-1} \leq \Gamma_{0} \leq 1 \times 10^{0}
$$$$
5 \times 10^{-3} \leq \Gamma_{0} \leq 2 \times 10^{-1}
$$

$1 \times 10^{-3} \leq \Gamma_{0} \leq 5 \times 10^{-3}$
Source Reynolds number

Plot Colour

Blue

Green

$900 \leq \operatorname{Re}_{0} \leq 2400$

Red

Table 1. Summary of source conditions for the experiments, in three bands of $\Gamma_{0}$ determined solely to aid the presentation of our data. The final column shows the colour that the data corresponding to each band of $\Gamma_{0}$ is plotted within figures 3-6.

in the range $2.0 \mathrm{~cm}^{3} / \mathrm{s} \leq Q_{0} \leq 7.5 \mathrm{~cm}^{3} / \mathrm{s}$ and the density of the saline solution varied between experiments to produce $14 \mathrm{~cm} / \mathrm{s}^{2} \leq g_{0}^{\prime} \leq 106 \mathrm{~cm} / \mathrm{s}^{2}$. Nozzles containing circular bores of radii $r_{0}=\{0.175 \mathrm{~cm}, 0.25 \mathrm{~cm}\}$ were used (for further details of the source conditions examined see appendix A). Given the moderate source Reynolds numbers $\left(\operatorname{Re}_{0}=M_{0}^{1 / 2} / \nu \sim 400\right.$, where $\nu$ denotes the kinematic viscosity), in some of our experiments, we consistently used plume nozzles which promoted turbulence within the flow by passing it through an expansion chamber and a narrow-gauge metal gauze prior to ejection. A summary of the plume source conditions is provided in table 1.

The visualisation tank was backlit by a slide projector (placed approximately $10 \mathrm{~m}$ from the tank) and shadowgraph images of the flow created by attaching a sheet of tracing paper to the side of the tank. Images were digitally recorded using a CCD camera (of approximately 1.5 Mpixels) at rates of 12 and 24 frames per second (i.e. less than standard video recording rates). Recording durations between $240 \mathrm{~s}$ and $2650 \mathrm{~s}$ provided datasets (of between 5500 and 32000 individual images) which were suitable for our analysis. Due to the limited volume of our tank and the required recording durations, a weak vertical flow was imposed within the ambient environment to avoid the tank filling up with saline solution. This was achieved by continually draining fluid from the saline layer which formed at the base of the tank and replacing this volume flux with a steady (low momentum) source of fresh water near the top of the tank. The imposed flow was such that our estimates of the vertical velocities imposed within ambient were always less than $4 \%$ of the local plume velocities. Full details of the experiments, including 
details of all source conditions and recording durations are provided in appendix A.

Reliably detecting the plume edges was a critical step in our analysis. Broadly speaking, this was possible since in our images the length scale at which molecular diffusion is expected to dominate, the Batchelor scale, was small (between $2 \times 10^{-5} \mathrm{~cm}$ and $6 \times 10^{-4} \mathrm{~cm}$ ) compared to one pixel $(0.02-0.05 \mathrm{~cm})$ but remains challenging due the nature of the billows at the plume edge that are advected and interact turbulently. To assist in the reliable detection of the plume edges we normalised all our experimental images by a background image (taken in the absence of any flow) and to gain confidence in the edges detected we employed two independent edgedetection algorithms. Our standard algorithm first overlaid edges onto the normalised image (these edges were identified using the Canny algorithm (Canny, 1986) provided within Matlab) and then identified the two plumes edges within each pixel row from the maximum (positive) and minimum (negative) horizontal light intensity gradient. Our alternate algorithm identified a threshold light intensity within each pixel row of each image and defined the plumes edges as the first and last location at which the light intensity fell below the threshold value - this threshold value was defined as the light intensity at which a minimum occurred, between the two peaks (corresponding to the presence and absence of plume fluid), in the histogram of light intensity within the given pixel row. For full details of the two algorithms see appendix B. Results of the plume edge locations from both algorithms can be seen in figure 1b. Also marked is the last pixel row at which the statistics for this particular plume were utilised — this height being the last pixel row for which the statistics from the two edge detection algorithms were sufficiently similar and the results could be deemed reliable. Figure $1 \mathrm{~b}$ shows that, above this height, the results from the two edge detection algorithms were practically identical.

From the horizontal distance between the locations of the plume edges we defined the local instantaneous plume half-width, $b(z, t)$, and time-averaging provided the plume half-width, $b_{T}=$ $b_{T}(z)$. Our measurements, neglecting those made very close to the source of plumes of $\Gamma_{0}<0.1$, exhibit a linear growth in the plume half-width $b_{T}$ with height $z$ and the gradient is independent of the source conditions. Applying a least-squares linear fit to the data for each plume, and 

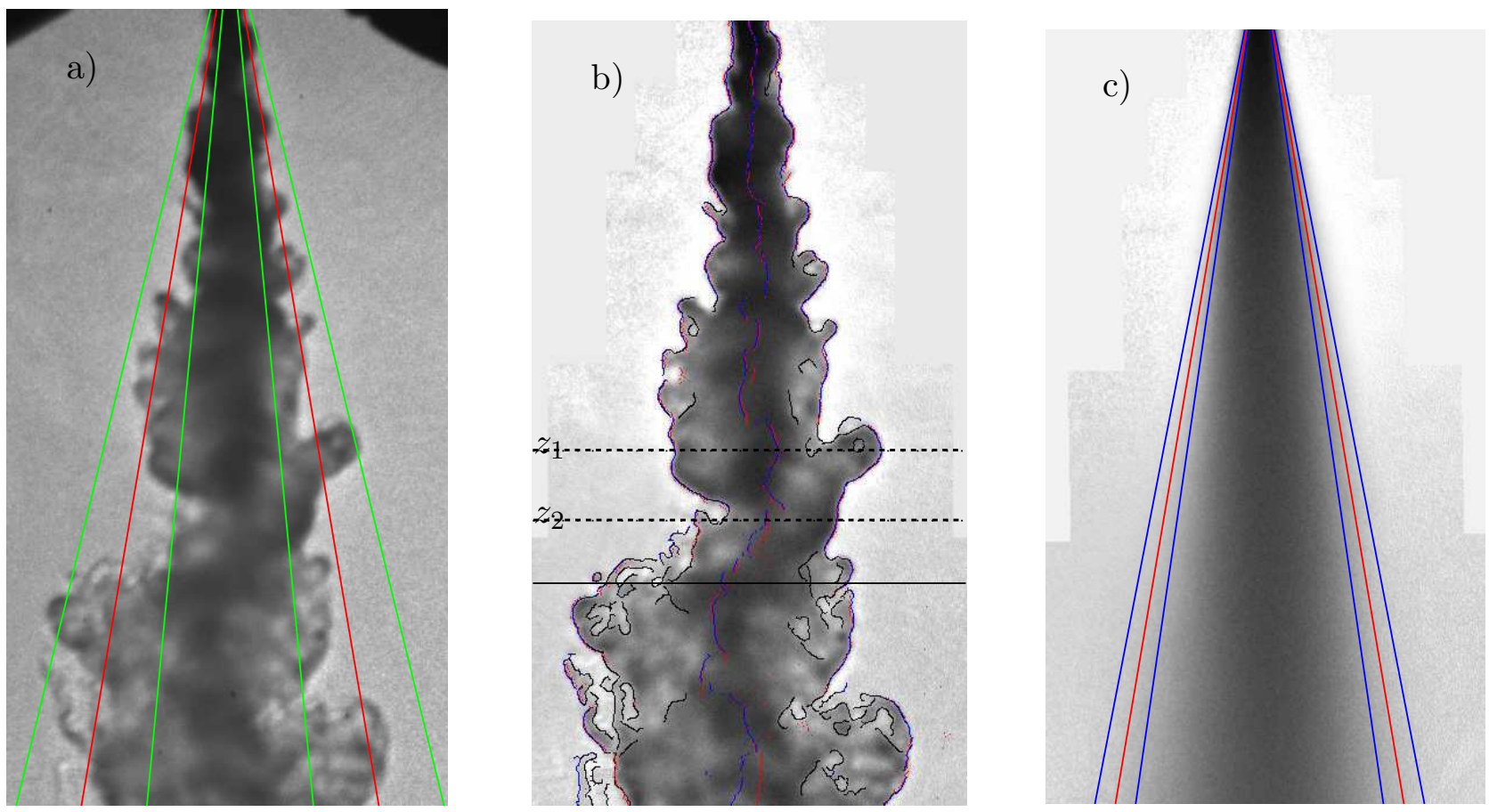

Fig. 1 a) An unprocessed instantaneous image of a plume with the scale of the mean width $2 b_{T}$ shown by the two red lines and the fluctuation scale $\delta b_{T}$ indicated by distance to the green lines either side. b) The associated processed image of the plume with instantaneous edges and centrelines marked, in red for the standard edge detection algorithm and blue for the alternate algorithm; the solid horizontal line marks the last pixel line for which reasonable statistics were obtained (see appendix B), the dotted lines mark the heights at which the times series in figure 2 were obtained. c) The time-averaged image of the plume with $b_{T}$ marked by red lines and the scale of the standard deviation in width indicated by blue lines.

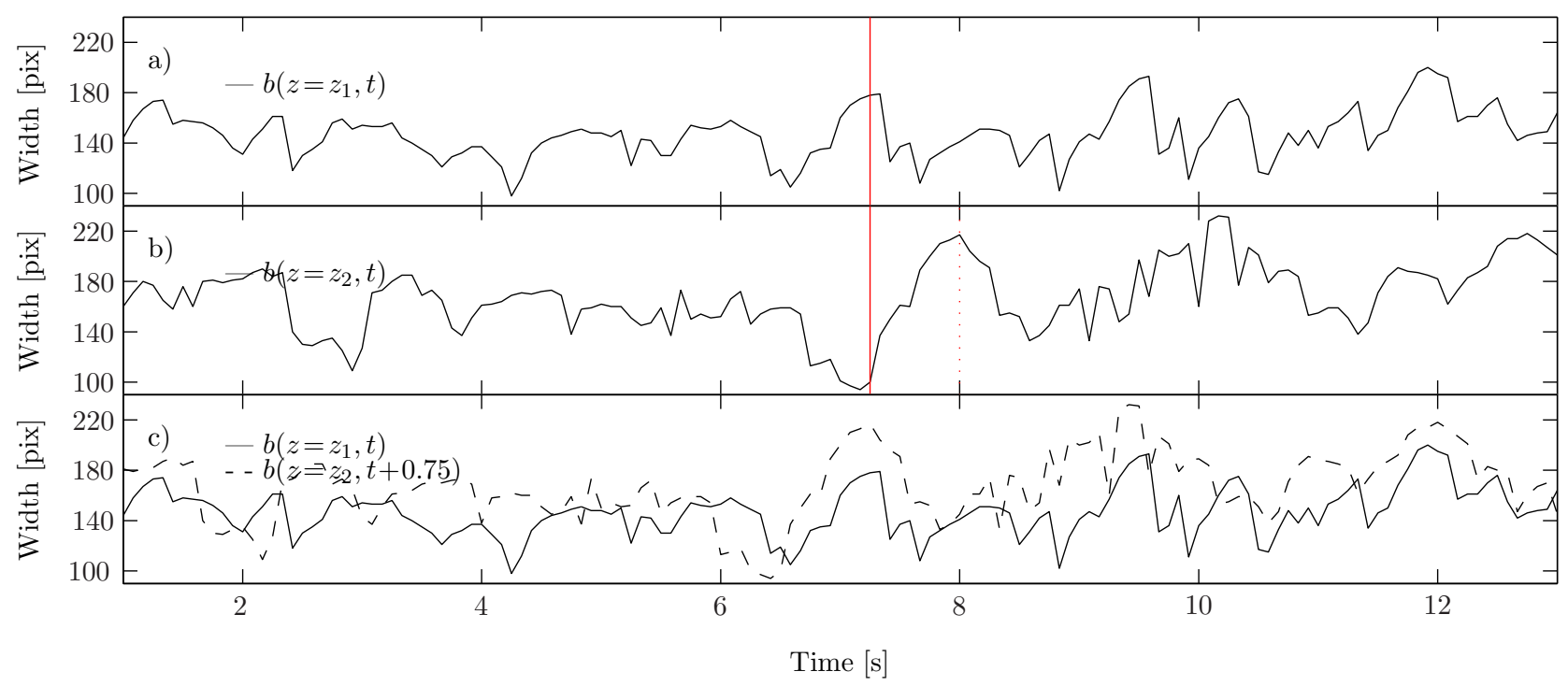

Fig. 2 Time series of plume half-width at the two heights, a) $b\left(z_{1}, t\right)$ and b) $b\left(z_{2}, t\right)$, marked by dotted lines in figure $1 \mathrm{~b}$.

The time, $t_{1}$, at which the image shown in figure $1 \mathrm{~b}$ was taken is marked by the solid red vertical line. The dotted red vertical line marks the time, $t_{1}+t_{c s}$, at which the cross-correlations indicate the coherent structure at the height $z_{1}$ within the image in figure $1 \mathrm{~b}$ would reach the height $z_{2}$. c) The two time series of plume half-width with one time series offset by $t_{c s}=0.75 \mathrm{~s}$. 
locating the point at which the fit predicts a plume half-width of zero, identified a vertical offset from the location of the physical source and defined the virtual origin, $z=0$, for each plume. The offset from the physical source, inferred for our experiments, varied depending on the relative forcing at the source, and was between $5 L_{Q}$ and $12 L_{Q}$ or, equivalently, $0.35 L_{M}$ and $2.1 L_{M}$.

We desired a measure for the magnitude of the fluctuations in plume width as large scale coherent structures (turbulent eddies) passed through. As an estimate for the scale of these fluctuations we defined measures reflective of the plume half-width in the presence, $b_{p}(z)$, and absence, $b_{a}(z)$, of large scale coherent structures. At a given height $z$, the width $b_{p}(z)$ was defined as the mean of all observations of $b(z, t)$ for which $b(z, t) \geq b_{T}+\sigma(z)$, where $\sigma(z)$ is the standard deviation of $b(z, t)$. The width $b_{a}(z)$ was defined as the mean of all observations for which $b(z, t) \leq b_{T}-\sigma(z)$. The fluctuation scale was then defined as the difference between these two widths, $\delta b_{T}=\delta b_{T}(z)=b_{p}(z)-b_{a}(z)$. Similar statistics have been shown to provide a meaningful indication of the scale of the fluctuations in other turbulent free-shear flows (Burridge \& Hunt, 2013). The ability of the statistic to characterise the scale of the fluctuations in plume width is indicated in figure 1a.

The peaks and troughs in the time series of plume width at a given height carry the signature of coherent structures passing through that height (figure 2). From the time series we were reliably able to detect the local vertical velocities of coherent structures at the plume edge $w_{c s}$. This was achieved by cross-correlating time series pairs from two different heights, the time lag which maximised the correlation provided an indication of the time $t_{c s}$ taken for coherent structures at the plume edge to travel between the two heights. An illustration of two time series at different heights, one of which is offset by the time $t_{c s}$ is shown in figure 2c. For each pair of heights, we calculated a number of estimates of the time lag by varying the starting point and duration of the time series examined at the two heights. We varied the starting point systematically between six discrete points within the recorded data and for each starting point we identified the lag which maximised the cross-correlations for eleven different length portions of the recorded data. The time taken for a coherent structure to travel between the two heights, 
$t_{c s}$, was then calculated as the mean of 66 lags identified by the cross-correlations of the data taken at the two heights (see appendix C). The gradient of $t_{c s}$, with the vertical coordinate $z$, enabled us to calculate a measure of the velocity of the coherent structures. In addition to the ensemble averaging within the values of $t_{c s}$, at a given height $z$ we evaluated the gradient of $t_{c s}$ within the data of the width at $z$ cross-correlated with data at a number of different heights (providing the heights were sufficiently close to height of interest $z$ that the correlations were reliable). Our estimates of the velocity of the coherent structures $w_{c s}=w_{c s}(z)$ were inferred from the mean local gradient $\mathrm{d} t_{c s} / \mathrm{d} z$ at $z$ taking eleven different observations - differing since one time series in the cross-correlation pair was taken at one of eleven different heights just above and below $z$ (see figure $\mathrm{C} 1$ in appendix $\mathrm{C}$ for an illustration). Figure 2 shows time series taken at two different heights within an experiment for which figure 1 shows images. For the example shown, the two heights are separated by a distance of approximately $4 L_{Q}$. Full details of the procedure are provided in appendix $\mathrm{C}$.

\section{Results}

Our measurements of the time-averaged half-width of the plumes, $b_{T}$, show a linear expansion for plumes with a gradient independent of $\Gamma_{0}$, the relative forcing at the source, for $z / L_{Q} \gtrsim 25$ or $z / L_{M} \gtrsim 1$ (figure 3a). This is consistent with the solutions of the conservation equations for a pure plume (Morton et al., 1956) and the gradient $\left(\mathrm{d} b / \mathrm{d} z=6 \alpha_{T} / 5\right)$ provides an estimate of the value of the entrainment coefficient of $\alpha_{T}=0.106 \sqrt{2}$ (marked by a solid black line in figure 3). Closer to the source, the forced plume widths $\left(\Gamma_{0} \lesssim 5 \times 10^{-3}\right)$ displayed in figure 3 show a marked departure from the linear expansion rate, supporting the notion that close to their source, forced plumes expand at a greater 'jet-like' rate $(\mathrm{d} b / \mathrm{d} z=2 \alpha)$. This is in agreement with the analysis of Morton (1959) (see figure 1 therein). However, that analysis predicts virtual origins for the forced plumes source conditions which are positioned behind those inferred from our experimental data (see $\S 2$ ). The precise reasons for this difference is unclear but may arise 

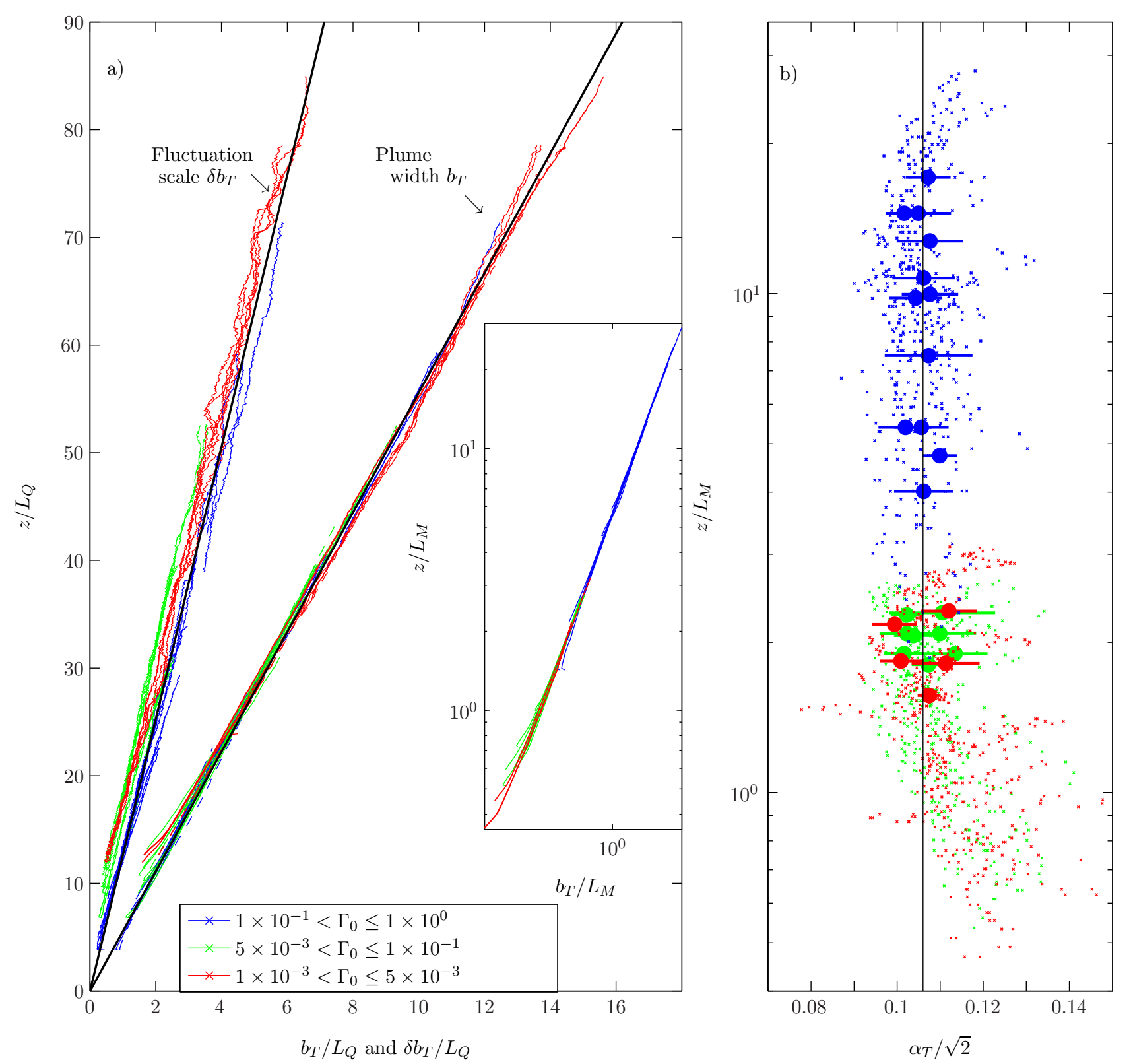

Fig. 3 a) The variation of the plume half-width and fluctuation scale with the vertical coordinate $z / L_{Q}$. The plume half-widths calculated with our alternate edge detection algorithm are marked by coloured dashed lines. The fits $b / z=$ $(6 / 5) 0.106 \sqrt{2}$ and $\delta b / z=0.44(6 / 5) 0.106 \sqrt{2}$ are marked by solid black lines, the inset shows the mean plume half-width scaled on $L_{M}$.

b) The variation of the entrainment coefficient $\alpha_{T}$ with the vertical coordinate scaled on the jet-length $L_{M}$ plotted on linear-log axes. The mean of $\alpha_{T}$ (for $z \geq 1.5 L_{M}$ ) from each experiment is marked by a circle with horizontal lines extending one standard deviation either side (the vertical position marks the mean height of measurement). The solid black vertical line marks $\alpha_{T}=0.106 \sqrt{2}$. 
from either the lack of a fully developed self-similar flow at the source in our experiments or a need to parameterise higher order 'turbulent' transport terms when modelling the near source fluxes of highly forced plumes. In figure 3 the plume half-widths determined by the alternate edge detection algorithm (see appendix B) are marked by dashed lines, the two different edge detection algorithms determine plume widths that are barely distinguishable from one another - providing confidence that the plume edges were reliably determined.

The closure model of Morton et al. (1956) required that the horizontal velocity of entrained fluid was equal to the product of a constant entrainment coefficient $\alpha_{T}$ and the local (tophat) vertical plume velocity $w_{T}$. Determining the precise value of the entrainment coefficient and its variation with the local plume parameter $\Gamma(z)$ is non-trivial and dedicated advanced experimental studies still continue (e.g. Ezzamel et al., 2015). Recent works tend to express the values for the entrainment coefficient appropriate on assuming Gaussian distributions for velocity and buoyancy within the plume, i.e $\alpha_{G} \equiv \alpha_{T} / \sqrt{2}$, and for clarity we explicitly include the factor $\sqrt{2}$ in the presentation of our data.

In part due to the reliable detection of the plume edges, our measurements provide a method for obtaining estimates of the entrainment coefficient by simply examining the gradient locally. Figure $3 \mathrm{~b}$ plots the variation of the entrainment coefficient $\alpha_{T}$ calculated from the local gradients of the plume half-widths (figure 3a). To highlight the variation with source forcing $\Gamma_{0}$, the data in figure $3 \mathrm{~b}$ is plotted with the vertical coordinate scaled on the jet-length, i.e. $z / L_{M}$. The data shows that $\alpha_{T}$ is approximately independent of $\Gamma_{0}$ for $z \gtrsim 1.5 L_{M}$ as supported by comparing values obtained for pure plumes with those from all of our plumes for $z \gtrsim 1.5 L_{M}$. The mean value, from all of the plumes irrespective of $\Gamma_{0}$, of $\alpha_{T}=(0.106 \pm 0.007) \sqrt{2}$ calculated from our gradient based data above $z=1.5 L_{M}$, closely matches with an alternate estimate of $\alpha_{T}=(0.106 \pm 0.002) \sqrt{2}$ based on the mean value of the scaled plume half-width, $b(z, t) /(6 / 5) z$, for only our data of $0.1 \leq \Gamma_{0} \leq 1$ (some $8.8 \times 10^{7}$ measurements). The tolerances reflect one standard deviation in our data. Our value for the entrainment coefficient is in good agreement with the findings from the latest numerical (Craske \& van Reeuwijk, Under consideration) and 
experimental (Ezzamel et al., 2015) investigations - a good summary of which is presented by van Reeuwijk \& Craske (2015).

For $z \lesssim 1.5 L_{M}$, we were only able to obtain data for forced plume sources (figure $6 \mathrm{~b}$ ) and the measurements of $\alpha_{T}$ show a noticeable bias above the mean. At first thought one may consider this to imply a greater entrainment coefficient to be applicable near the source of the forced plumes. However, the values of $\alpha_{T}$ are calculated assuming that $\mathrm{d} b_{T} / \mathrm{d} z=6 \alpha_{T} / 5$, i.e. a plumelike behaviour, while in the near field of a highly forced plume we expect $\mathrm{d} b_{T} / \mathrm{d} z=2 \alpha_{J}$, where $\alpha_{J}$ denotes the entrainment coefficient appropriate for a jet-like flow. Taking the mean over our highly forced plume data for $z \leq 1.0 L_{M}$ and assuming jet-like behaviour gives an estimate $\alpha_{J} \approx 0.065 \sqrt{2}$ - this value is consistent with a reduction of the entrainment coefficient expected for jet-like flows relative to plumes.

Figure $3 \mathrm{a}$ also shows the characteristic scale $\delta b_{T}$ of the fluctuations at the plume edge (see $\S 2)$. From our data, we cannot identify any systemic variation in the fluctuation scale with the source forcing $\Gamma_{0}$. The fluctuation scale $\delta b_{T}$ exhibits a linear increase with distance from the source, the gradient of which is approximately $44 \%$ that of the mean plume width. Given that $\delta b_{T}$ is representative of the magnitude of the turbulent billows at the plume edge, $\delta b_{T} \propto b_{T} \propto z$ suggests that both the scale of turbulent eddies and the mean plume width evolve similarly. For comparison a graphical representation of the ability of the statistic $\delta b_{T}$ to indicate a characteristic scale for the fluctuations is provided in figure $1 \mathrm{a}$ in which the mean plume width $b_{T}$ is overlaid (in red) on an instantaneous image of a plume. Two (green) lines provide an indication of our fluctuation scale at both edges of the plume - the fluctuation scale can be seen to be broadly representative of the magnitude of the turbulent billows at the plume edge. 

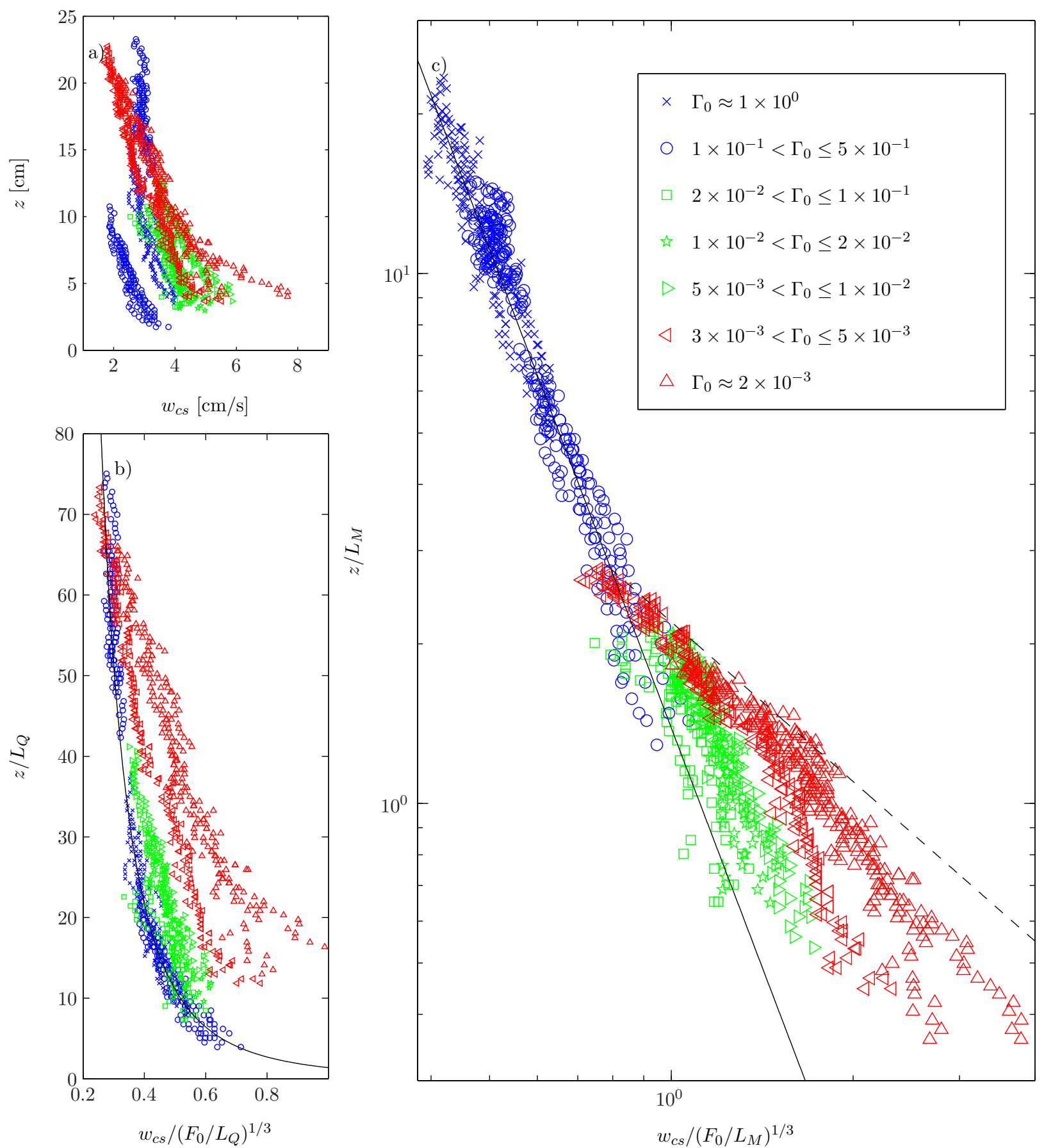

Fig. 4 The variation in the velocity of coherent structures at the plume edge $w_{c s}$ with the vertical coordinate. a) The unscaled data, b) the data scaled with the source scale $L_{Q}$ and c) the data on log-log axes scaled with the jet-length $L_{M}$. The solid black lines in b) and c) mark the best-fit (6). The dashed black line in c) marks the power-law expected for jet-like behaviour, i.e. $w \propto z^{-1}$.

The velocities of the coherent structures at the plume edge $w_{c s}$ are plotted in figure 4 . The scaled data for relatively pure plumes exhibit the expected decay in the velocity, $w_{c s} \propto z^{-1 / 3}$ 
(figures $4 \mathrm{~b}$ and c). This decay is supported over the entire vertical extent for which we were able to capture data. The data for highly forced plumes, at least over $1 \lesssim z / L_{M} \lesssim 3$, approximately follow a $w_{c s} \propto z^{-1}$ decay (figure $4 \mathrm{c}$ ) — the scaling expected for the mean velocities in jet-like flows. These findings indicate that the velocities of coherent structures (turbulent eddies) at the edge are inherently coupled with the characteristic time-averaged velocities within the flow.

Closer to the source the velocities in highly forced plumes decay less rapidly, perhaps an indication that for $z \ll L_{M}$ the flow was not fully developed. It is regrettable that, due to the physical limitations of our experiments we were not able to obtain reliable data for highly forced plumes further from the source, i.e $z \gtrsim 3 L_{M}$. As such it is not entirely surprising that our highly forced data do not show any decay which could be regarded as plume-like. However, our more moderately forced plume data do show evidence of plume-like behaviour. The distance from the source required to detect this behaviour is itself a function of the forcing at the source. For plumes with $2 \times 10^{-2}<\Gamma_{0} \leq 1 \times 10^{-1}$, the velocities closely match those of pure plumes for $0.6 \lesssim z / L_{M} \lesssim 2$ (figure $4 \mathrm{~b}$ and c). For some lower $\Gamma_{0}$ plumes, a plume-like decay in the velocities is evident but only for $z \gtrsim 1.5 L_{M}$, for which the data are limited; we return to the behaviour of these forced plumes at the end of this section.

For relatively pure plumes, taking $\alpha_{T}=0.106 \sqrt{2}$, we obtain a reasonable fit (plotted in figures $4 \mathrm{~b}$ and $4 \mathrm{c}$, coefficient of determination of $R^{2}=93.6 \%$ ) for the velocity of the coherent structures by applying a constant pre-factor to the top-hat plume velocities predicted by Morton et al. (1956), i.e.

$$
w_{c s}=0.57 \times\left(5 / 6 \alpha_{T}\right)\left(9 \alpha_{T} / 10 \pi\right)^{1 / 3} F_{0}^{1 / 3} z^{-1 / 3}=0.57 w_{T} .
$$

This indicates that the coherent structures at the edge of a pure plume travel at almost $60 \%$ of the local top-hat plume velocity. A physical interpretation can be provided by comparison to time-averaged centreline velocities which are not affected by spatial intermittency as the plume meanders (Papantoniou \& List, 1989) and, therefore, provide more representative characteristic velocities. Plume centreline velocities have been measured using more advanced experimental 
techniques than in the present study (e.g. Ezzamel et al., 2015; Shabbir \& George, 1994). These studies report a good fit of the velocities to a Gaussian model for the plume velocity, i.e. the characteristic plume velocity is $w_{G}=2 w_{T}$. Hence the coherent structures at the plume edge travel at approximately $30 \%$ the characteristic (centreline) velocity, i.e. $w_{c s} \approx 0.3 w_{G}$. Some reassurance might be taken from such a value when one considers that the process of turbulent entrainment (at least the large-scale engulfment of ambient fluid) is likely to be driven by the coherent eddying structures at the plume edge. With the characteristic horizontal entrainment velocity being $u_{E}=\alpha_{G} w_{G}$ with $\alpha_{G} \approx 0.1$, one can then envisage a cascade of velocities from the characteristic plume velocity to the vertical velocity at the plume edge drawing in ambient fluid and driving a (predominantly) horizontal flow in the ambient, i.e. $w_{G}>w_{c s} \approx 0.3 w_{G}>$ $u_{E} \approx 0.3 w_{c s}$.

From the measurements of the (top-hat) plume half-widths $b_{T}$ and velocity $w_{T}$ (based on measurements of $w_{c s}$ ) we plot estimates for the evolution of the volume flux in figure 5a, along with the volume flux expected for a point source pure plume, $\mathrm{E}[Q]=$ $\left(6 \alpha_{T} / 5\right)\left(9 \alpha_{T} / 10\right)^{1 / 3} \pi^{2 / 3} F_{0}^{1 / 3} z^{5 / 3}$ - our data for relatively pure plumes show good agreement with the theoretical predictions. The mean value of our estimate of the plume volume flux normalised by the theoretical predictions is $Q(z) / \mathrm{E}[Q]=1.01 \pm 0.04$ for relatively pure plumes, and $Q(z) / \mathrm{E}[Q]=1.04 \pm 0.08$ for all plumes of $5 \times 10^{-3} \leq \Gamma_{0} \leq 1$. This indicates that even for highly forced sources the analysis predicts the volume flux accurately to within about $10 \%$ and for pure plumes to within approximately $5 \%$. Figure $5 \mathrm{~b}$ plots the equivalent data for the momentum flux $M(z)$ - these data exhibit similar trends. Throughout their vertical extent, the highly forced plumes exhibit an excess of volume (mass) and momentum relative to pure plumes consistent with expectations (Morton, 1959).

The conservation equations (2) allow the buoyancy flux to be calculated from the spatial derivative of the momentum flux and the local plume velocity

$$
F=\frac{M}{Q} \frac{\mathrm{d} M}{\mathrm{~d} z}=\pi b_{T}^{2} w_{T} g_{T}^{\prime}
$$




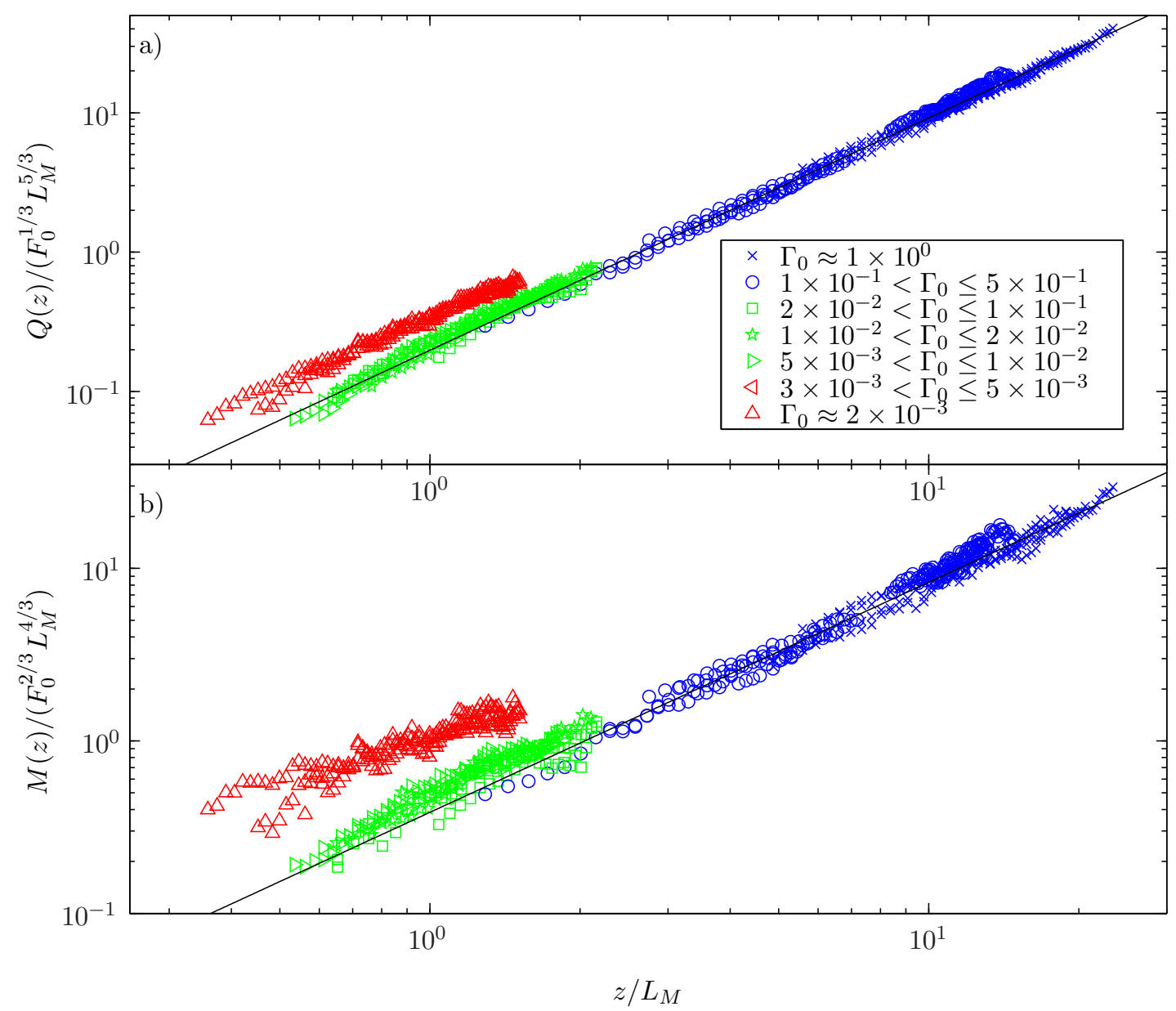

Fig. 5 The variation in estimated a) plume volume flux $Q(z)=\pi b_{T}^{2} w_{T}$ and b) momentum flux $M(z)=\pi b_{T}^{2} w_{T}^{2}$ with the vertical coordinate scaled on the jet-length. The solid black line in a) mark $Q=\left(6 \alpha_{T} / 5\right)\left(9 \alpha_{T} / 10\right)^{1 / 3} \pi^{2 / 3} F_{0}^{1 / 3} z^{5 / 3}$ and in b) $M=\left(6 \alpha_{T} / 5\right)^{4 / 3}\left(5 / 8 \alpha_{T}\right)^{2 / 3} \pi^{1 / 3} F_{0}^{2 / 3} z^{4 / 3}$ both with $\alpha_{T}=0.106 \sqrt{2}$.

Since the data for relatively pure plumes exhibit a good agreement with theoretical predictions we are able to calculate estimates for $\mathrm{d} M / \mathrm{d} z$ by fitting (in a least-squares sense) a power-law of the form $M \propto z^{4 / 3}$ (figure 5b). Differentiating this fit, combined with our estimates of the velocity $w_{T}=w_{c s} / 0.57(6)$, provides predictions of the local buoyancy flux for each of the relatively pure plumes.

For the more forced plumes the fit of the momentum flux to the predicted power-law in $z$ for a pure plume is understandably less good. In order to provide estimates of the buoyancy flux in these plumes, from $\mathrm{d} M / \mathrm{d} z$, we obtain the best fit to the momentum flux data to power-laws, $M \propto z^{p}$. We determine both the constant of proportionality and the exponent $p$ by locally fitting the data for each plume over 25 data points (equivalent to between $0.2 L_{M}$ and $0.4 L_{M}$ ) i.e. we 
allow the power-law for the momentum flux to evolve with distance from the source. Again differentiating these fits, and combining with estimates of $w_{T}$, provides predictions of the local buoyancy flux for each plume.

The results for the local buoyancy flux are particularly informative since it is conserved for each plume and we have reliable measurements of the source buoyancy flux, $F_{0} \equiv Q_{0} g_{0}^{\prime}$. We present our data for the normalised local buoyancy flux in figure 6a. For relatively pure plume sources, there is no bias exhibited resulting from the measurement location and the mean of the estimates is $F(z) / F_{0}=1.04 \pm 0.15$, the tolerance being one standard deviation. Hence, the estimates prove to be good (within approximately 15\%) at predicting the true buoyancy flux when one considers that the estimates of $F(z)$ are made entirely from videos of a plume without knowledge of the source conditions or the location of the source (note that we inferred the virtual origins based on measurements of $\mathrm{d} b_{T} / \mathrm{d} z$ see $\S 2$ ).

For forced plumes the estimates overpredict the buoyancy flux. However, the data show that this overprediction decreases with distance from the source, presumably as the balance of inertia and buoyancy within the plume approaches that of a pure plume (see figure 6b). For example, the data furthest from the source that we were able to gather, $z \approx 2 L_{M}$, for moderately forced plumes provide a reasonable estimate of the buoyancy flux, $F(z) / F_{0}=1.13 \pm 0.19$. Hence, irrespective of $\Gamma_{0}$, we expect to be able to predict the buoyancy flux in a plume within a uniform environment with reasonable $( \pm 19 \%)$ accuracy, based on solely on images of a section of the plume provided that the images are taken appropriate distances from the source $\left(z \gtrsim 2 L_{M}\right)$.

\section{Implications and conclusions}

This study has provided a robust estimate for the entrainment coefficient required for the closure of plume models following Morton et al. (1956). For heights $z \gtrsim 1.5 L_{M}$ the entrainment coefficient is invariant with source conditions (at least for $0.001 \leq \Gamma_{0} \leq 1$ ) and a value of $\alpha_{T}=(0.106 \pm 0.007) \sqrt{2}$, is broadly appropriate. 


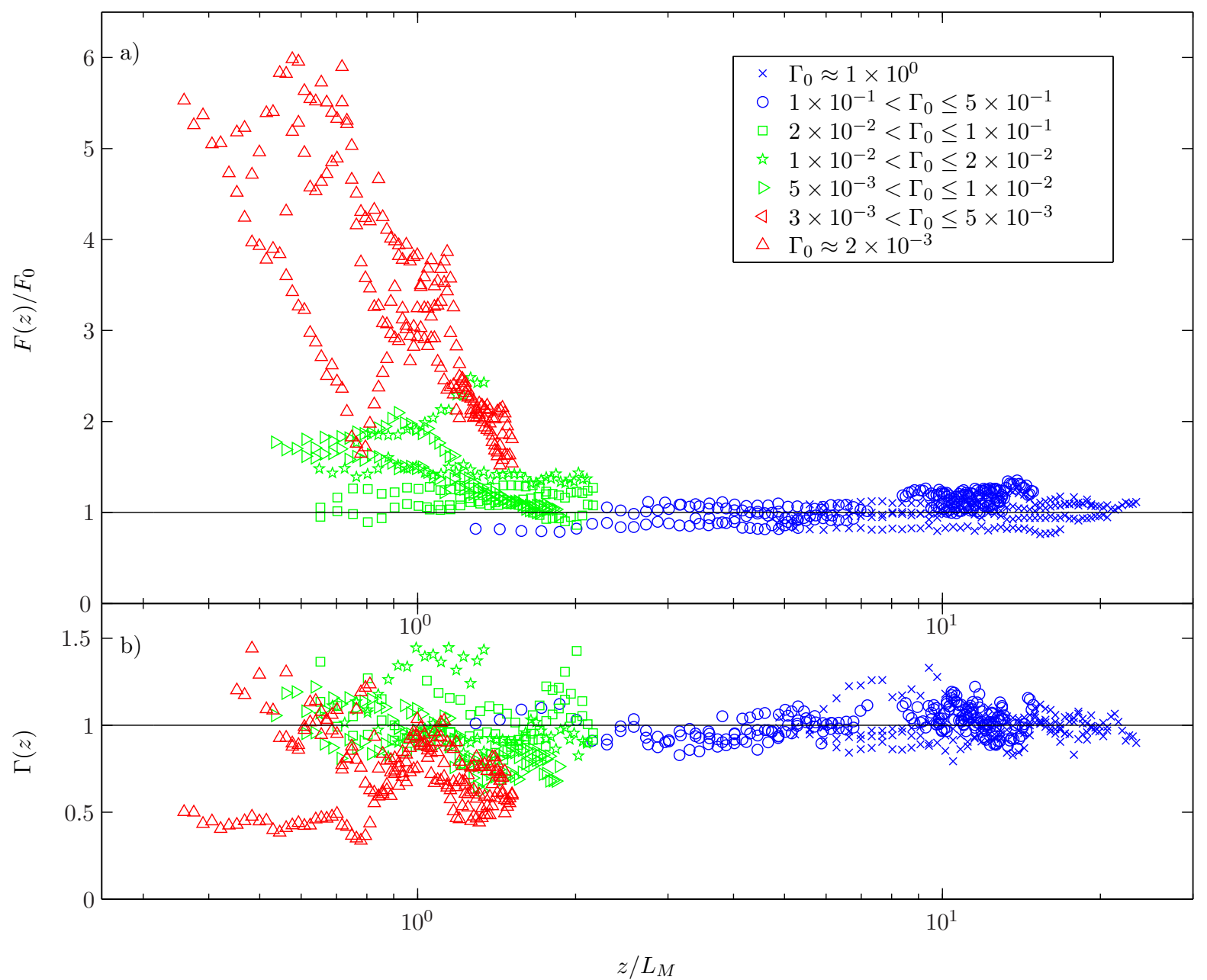

Fig. 6 The variation with the vertical coordinate (scaled on the jet-length) of a) the estimated plume buoyancy flux $F(z)=\pi b_{T}^{2} w_{T} g_{T}^{\prime}$ normalised on the (conserved) source buoyancy flux and b) the plume parameter $\Gamma$. The solid black line in a) marks $F(z)=F_{0}$ and in b) $\Gamma=1$.

The measurements presented for the velocity of structures at the plume edge $w_{c s}$ were not time-averaged, since by cross-correlating the signatures of plume width we are ensemble averaging the evolution of numerous turbulent eddies which remain coherent at the plume edge over some vertical extent. As such, they are informative as to the structure of the instantaneous velocity distribution within the plume and suggest that the vertical velocities at the plume edge are of the same order as the top-hat or centreline velocities. Furthermore, $w_{c s}$ may be regarded as a measurement of a turbulent velocity scale. Our results suggest that both the spatial extent $\delta b_{T}$ and velocity $w_{c s}$ of the turbulent structures at the plume edge are directly proportional to 
the time-averaged width and velocity of the plume. This provides some insight as to how the description of a plume in terms of only the mean quantities by Morton et al. (1956) is able to capture the dynamics of such a complex turbulent flow.

The analysis of the plume widths deduced within movie images of the bulk flow has enabled the fluxes of volume (mass), momentum and buoyancy to be estimated. Estimates of the volume flux in a plume are of real consequence. For example, estimates of the volume flux in the plume emanating from a severed riser tube were used to asses the scale of the Deepwater horizon oil leak (McNutt, Camilli, Crone, Guthrie, Hsieh, Ryerson, Savas \& Shaffer, 2012) and in that case movie images were available. Our estimates for the volume flux in relatively pure plumes agree with theoretical predictions to within approximately 5\%, and our estimates for forced plumes can be expected to be similarly accurate providing that the radial distributions of velocity are similar in pure and forced plumes, i.e. the velocity of coherent structures at the edge are approximately $60 \%$ of the top-hat velocities, or $30 \%$ of the (Gaussian) plume centreline velocities. As such, the analysis may be regarded as providing estimates for the volume flux in a plume with unprecedented accuracy; estimates which can be made, irrespective of the source conditions, based on data readily available in the field. It is worth noting that we have not examined the effects that motion within the ambient environment (e.g. winds or currents) might have on our analysis. In most situations for which the motion within the ambient environment is significant the tracking of coherent eddies at the plume edge does not present any fundamental issues. An exception to this being cases for which local asymmetries within the ambient conditions give rise to a significant swirl within the plume (as is the case within fire whirls).

Our estimates of the buoyancy flux in relatively pure plumes were accurate to within approximately $15 \%$ - the buoyancy flux being critical in determining the evolution for real plumes. For example, for volcanic plumes rising in the atmosphere the source buoyancy flux is typically estimated by taking a characteristic value for the buoyancy (or Brunt Väisälä) frequency and observing the height at which plume fluid intrudes and spreads radially. Where such observations are more challenging or not possible, for example in the ocean, our analysis may provide an al- 
ternate method for estimating the buoyancy flux and spreading height of the plume. In order to apply our analysis to a plume rising in a stratified environment one would either need to account for the change in the buoyancy flux with height due to the stratification or examine the plume over a vertical region which is sufficiently small that the changes in the buoyancy flux are relatively small (e.g. of the same order as the uncertainty). In the atmosphere and oceans the buoyancy frequency is approximately $N=O\left(10^{-2} \mathrm{~s}^{-1}\right)$. From the plume conservation equations in a stratified environment $(\mathrm{d} F / \mathrm{d} z) / F \propto N^{2} / g^{\prime}$. Taking the normalised density difference between the plume and the environment to be in the range $0.1 \%$ to $1 \%$, i.e. $g^{\prime}=O\left(10^{-1}-10^{-2} \mathrm{~m} / \mathrm{s}^{2}\right)$, provides that the buoyancy flux changes by an amount $\delta F=O\left(10^{-2}-10^{-3} F\right)$ per vertical metre travelled. Therefore, if one carries out our analysis on a plume in the atmosphere or ocean, then basing the analysis on coherent structures at the plume edge evolving of over a height of, say, $10 \mathrm{~m}$, the error that one introduces on assuming the buoyancy flux is constant over that height would be in the range 1\%-10\% and comparable to the uncertainty within the analysis. Therefore it may be appropriate, and indeed useful, to directly apply our analysis to estimate the buoyancy flux of plumes within the atmosphere or ocean.

The authors would like to acknowledge that the genesis of the work reported herein was a study investigating the physics of turbulent fountains, via coherent structure tracking, which was carried out by HCB and Prof. Gary R. Hunt (Burridge \& Hunt, In Preparation). In addition, the authors gratefully acknowledge the skills and expertise provided by the technical staff at the G. K. Batchelor laboratory; HCB acknowledges the insightful comments of Prof. Colm-cille Caulfield. This work was supported, in part, by the Leverhulme Trust Research Programme Grant RP2013-SL-008, the EPSRC Programme Grant EP/K034529/1 and by the Royal Society. 


\section{Appendix A. Experimental details}

Plumes were created by steadily ejecting dyed saline solutions downwards from circular bore nozzles of radii $r_{0}=\{0.175 \mathrm{~cm}, 0.25 \mathrm{~cm}\}$. In order to promote turbulence within the flow near the source, plume nozzles were used which pass the flow through an expansion chamber and a narrowgauge metal gauze prior to ejection. The resulting flow appeared turbulent indistinguishably close to the source even at some moderate source Reynolds numbers. For three experiments (marked by the symbol ${ }^{\dagger}$ in table A1) the flow only appeared turbulent 5-10 source diameters downstream of the source and data above this height have herein been excluded. These were not our lowest $\mathrm{Re}_{0}$ experiments but were the lowest $\mathrm{Re}_{0}$ experiments produced with the smaller bore nozzle, i.e. $r_{0}=0.175 \mathrm{~cm}-$ this indicates that $\mathrm{Re}_{0}$ is not entirely representative of the level of turbulence at the source when a plume nozzle is utilised.

The plume nozzles were rigidly clamped and hung (approximately $25 \mathrm{~cm}$ from the freesurface) within a glass sided visualisation tank, of cross-section $80 \mathrm{~cm} \times 100 \mathrm{~cm}$ filled to a depth of $90 \mathrm{~cm}$ with fresh water. The flow was driven by a constant (gravitational) head apparatus and the flow rate controlled and measured using a needle valve and variable area flowmeter, respectively. By setting the source volume fluxes, in the range $2.0 \mathrm{~cm}^{3} / \mathrm{s} \leq Q_{0} \leq 7.5 \mathrm{~cm}^{3} / \mathrm{s}$, and the measuring the densities of the saline solution and freshwater environment using an Anton Paar densitometer to calculate the buoyancy, in the range $14 \mathrm{~cm} / \mathrm{s}^{2} \leq g_{0}^{\prime} \leq 7.5 \mathrm{~cm} / \mathrm{s}^{2}$, the buoyancy flux at the source was known to an accuracy of approximately $2 \%$. See table A1 for full details of all the experimental conditions used.

The visualisation tank was backlit by a slide projector (placed approximately $10 \mathrm{~m}$ from the tank) to create shadowgraph images of the flow on a sheet of tracing film attached to the side of the tank. To further aid visualisation, the fluid (solutions of $\mathrm{NaCl}$ in fresh water) ejected to form the plume was stained with a colour dye. The molecular diffusivity of the dye was broadly similar to that of $\mathrm{NaCl}$ so that Schmidt numbers (the ratio of viscous and molecular diffusivities) were high and, even at moderate Reynolds numbers, the flow was always high Péclet number. 


\begin{tabular}{|c|c|c|c|c|c|c|c|}
\hline & $\begin{array}{c}Q_{0} \\
{\left[\mathrm{~cm}^{3} / \mathrm{s}\right]}\end{array}$ & $\begin{array}{c}g_{0}^{\prime} \\
{\left[\mathrm{cm} / \mathrm{s}^{2}\right]}\end{array}$ & $\begin{array}{c}r_{0} \\
{[\mathrm{~cm}]}\end{array}$ & $\Gamma_{0}$ & $\operatorname{Re}_{0}$ & $\begin{array}{c}\text { Frame Rate } \\
\qquad[1 / \mathrm{s}]\end{array}$ & Images \\
\hline Pure & 2.0 & 89.6689 & 0.250 & $9.5 \times 10^{-1}$ & 451 & 12 & 23,668 \\
\hline Pure & 2.0 & 89.6689 & 0.250 & $9.5 \times 10^{-1}$ & 451 & 12 & 8,890 \\
\hline Pure & 2.0 & 89.6689 & 0.250 & $9.5 \times 10^{-1}$ & 451 & 12 & 18,000 \\
\hline Pure & 2.0 & 89.6689 & 0.250 & $9.5 \times 10^{-1}$ & 451 & 12 & 14,767 \\
\hline Relatively Pure & 2.0 & 32.9037 & 0.250 & $3.5 \times 10^{-1}$ & 451 & 12 & 31,567 \\
\hline Relatively Pure & 2.0 & 32.9037 & 0.250 & $3.5 \times 10^{-1}$ & 451 & 12 & 5,000 \\
\hline Relatively Pure & 2.0 & 32.9037 & 0.250 & $3.5 \times 10^{-1}$ & 451 & 12 & 10,501 \\
\hline Relatively Pure & 2.4 & 21.7679 & 0.250 & $1.6 \times 10^{-1}$ & 542 & 12 & 8,767 \\
\hline Relatively Pure & 2.4 & 21.7679 & 0.250 & $1.6 \times 10^{-1}$ & 542 & 12 & 24,000 \\
\hline Relatively Pure ${ }^{\dagger}$ & 2.7 & 106.6063 & 0.175 & $1.0 \times 10^{-1}$ & 881 & 12 & $3,767^{*}$ \\
\hline Relatively Pure ${ }^{\dagger}$ & 2.7 & 106.6063 & 0.175 & $1.0 \times 10^{-1}$ & 881 & 24 & 21,283 \\
\hline Relatively Pure $^{\dagger}$ & 2.7 & 106.6063 & 0.175 & $1.0 \times 10^{-1}$ & 881 & 24 & 5,501 \\
\hline Moderately forced & 7.5 & 31.0654 & 0.250 & $2.4 \times 10^{-2}$ & 1,693 & 12 & 24,001 \\
\hline Moderately forced & 7.5 & 26.0837 & 0.250 & $2.0 \times 10^{-2}$ & 1,693 & 12 & 13,428 \\
\hline Moderately forced & 7.5 & 26.0837 & 0.250 & $2.0 \times 10^{-2}$ & 1,693 & 12 & 11,253 \\
\hline Moderately forced & 4.8 & 37.5642 & 0.175 & $1.1 \times 10^{-2}$ & 1,563 & 12 & 11,000 \\
\hline Moderately forced & 4.8 & 37.5642 & 0.175 & $1.1 \times 10^{-2}$ & 1,563 & 24 & 31,201 \\
\hline Moderately forced & 4.8 & 37.5642 & 0.175 & $1.1 \times 10^{-2}$ & 1,563 & 12 & 16,000 \\
\hline Moderately forced & 7.3 & 37.5642 & 0.175 & $5.1 \times 10^{-3}$ & 2,344 & 12 & 13,001 \\
\hline Moderately forced & 7.3 & 37.5642 & 0.175 & $5.1 \times 10^{-3}$ & 2,344 & 12 & 16,767 \\
\hline Highly forced & 6.1 & 18.2288 & 0.175 & $3.6 \times 10^{-3}$ & 1,954 & 12 & 15,664 \\
\hline Highly forced & 6.1 & 18.2288 & 0.175 & $3.6 \times 10^{-3}$ & 1,954 & 12 & 15,664 \\
\hline Highly forced & 7.3 & 14.0789 & 0.175 & $1.9 \times 10^{-3}$ & 2,344 & 24 & 12,000 \\
\hline Highly forced & 7.3 & 14.0789 & 0.175 & $1.9 \times 10^{-3}$ & 2,344 & 24 & 5,742 \\
\hline Highly forced & 7.3 & 14.0789 & 0.175 & $1.9 \times 10^{-3}$ & 2,344 & 24 & 20,767 \\
\hline
\end{tabular}

Table A1. The source conditions for all of our experiments with details of the recordings made. The three experiments marked ${ }^{\dagger}$ appeared turbulent only after approximately $5-10$ source diameters and the data above this height was excluded, all other experiments appeared turbulent at the source. The number of images marked ${ }^{*}$ highlights that the statistics obtained from this experiment were insufficient to obtain reliable measurements of the velocity $w_{c s}$.

.


The dye therefore accurately marked the location of the plume fluid; plume fluid referring to all fluid with a density that had been affected by the presence of the saline source. Digital images, $1024 \times 1372$ pixels, were recorded using a JAI CVM4 ${ }^{+}$CL CCD camera at rates of 12 and 24 frames per second and stored as 8-bit image files, i.e. the light intensity for each pixel was stored with a value between 0 (pure black) and 255 (pure white). Recording durations were between approximately $240 \mathrm{~s}$ and $2650 \mathrm{~s}$ (see table A1). The resulting datasets, of between 3767 and 31567 images, were then analysed. Data for the velocity $w_{c s}$ and hence the fluxes could only be determined from datasets larger than 5500 images.

During the course of our experiments a layer of (saline) plume fluid accumulated at the base of the tank. Given the volume of our tank and the desired recording durations, to avoid plume fluid filling the entire tank the saline plume fluid was steadily drained from the base of the tank. To maintain the free surface at a constant height within the tank, the volume flux being drained was replaced by a steady source of fresh water near the top of the tank. The incoming fresh water was passed though a number of layers of porous foam in order to diffuse a sufficient portion of the incoming momentum. For our experiments the draining rate was not required to be carefully measured but was typically between approximately $300 \mathrm{~cm}^{3} / \mathrm{s}$ and $750 \mathrm{~cm}^{3} / \mathrm{s}$. As a consequence of the continual draining and replacing of fluid a weak vertical flow was imposed within the ambient environment. From knowledge of the cross-sectional area of the tank, measurements of the draining rate, and estimates of plume volume flux and cross-sectional area, we calculate that the velocities of the imposed flow were always less than $4 \%$ of the local plume velocities.

\section{Appendix B. Determining the plume edges}

The analysis centred on reliably defining and detecting the plume edges over some reasonable portion of each image. To achieve this, we first normalised each image using a background image taken in the absence of the plume. Reliably detecting the plume edges was physically achievable since the Péclet number of our plumes were large and the scale at which molecular mixing 
and diffusion are expected to dominate, the Batchelor scale, was small (between $2 \times 10^{-5} \mathrm{~cm}$ and $\left.6 \times 10^{-4} \mathrm{~cm}\right)$ compared with the size of a pixel $(0.02-0.05 \mathrm{~cm}$ in our experiments). Hence with suitable algorithms one could expect to resolve the plume edge to within one pixel. For reassurance we wrote two edge detection algorithms which both aimed to detect the edges in an independent manner, we refer to these as the standard algorithm and the alternate algorithm.

The standard algorithm first executed a subroutine consisting of the Canny algorithm (Canny, 1986) provided within Matlab on each background normalised image. The Canny algorithm first applies a local Gaussian filter slightly blurring the image (reducing noise and minimising the effects of discretisation within the image) and then identifies edges by locating local maximums in the light intensity gradients within the filtered image — these edges are then sharpened using local non-maximum suppression. 'Strong' edges within the image are determined where the local maximum gradients exceed a high threshold value and a larger number of 'weak' edges where the gradients exceed a lower threshold value. The Canny algorithm finally returns an image including all the strong edges identified combined with any weak edges conditional that the selected weak edges are attached to a strong edge. Within our normalised image we set the light intensity to zero (pure black) at all locations at which the Canny algorithm identified an edge. Within each pixel row our standard algorithm then identified the locations of the minimum (negative) and maximum (positive) light intensity gradients within the pixel row and stored these locations as the left and right hand plume edges, respectively. The locations of the two edges were stored for each row and the algorithm was then repeated on the next image.

The alternate algorithm worked quite differently. For each row of pixels within an image the alternate algorithm examined the distribution of light intensities typically identifying a bimodal distribution of light intensity with the two peaks corresponding to regions where plume fluid is present and absent. Within a pixel row, for example, in regions where plume fluid is absent the pixels (within the background normalised image) exhibit high values of light intensity (typically relatively near saturation). Between these two peaks, the algorithm identifies the value of the light intensity at which there is a minimum in the distribution, the value of the light intensity at 


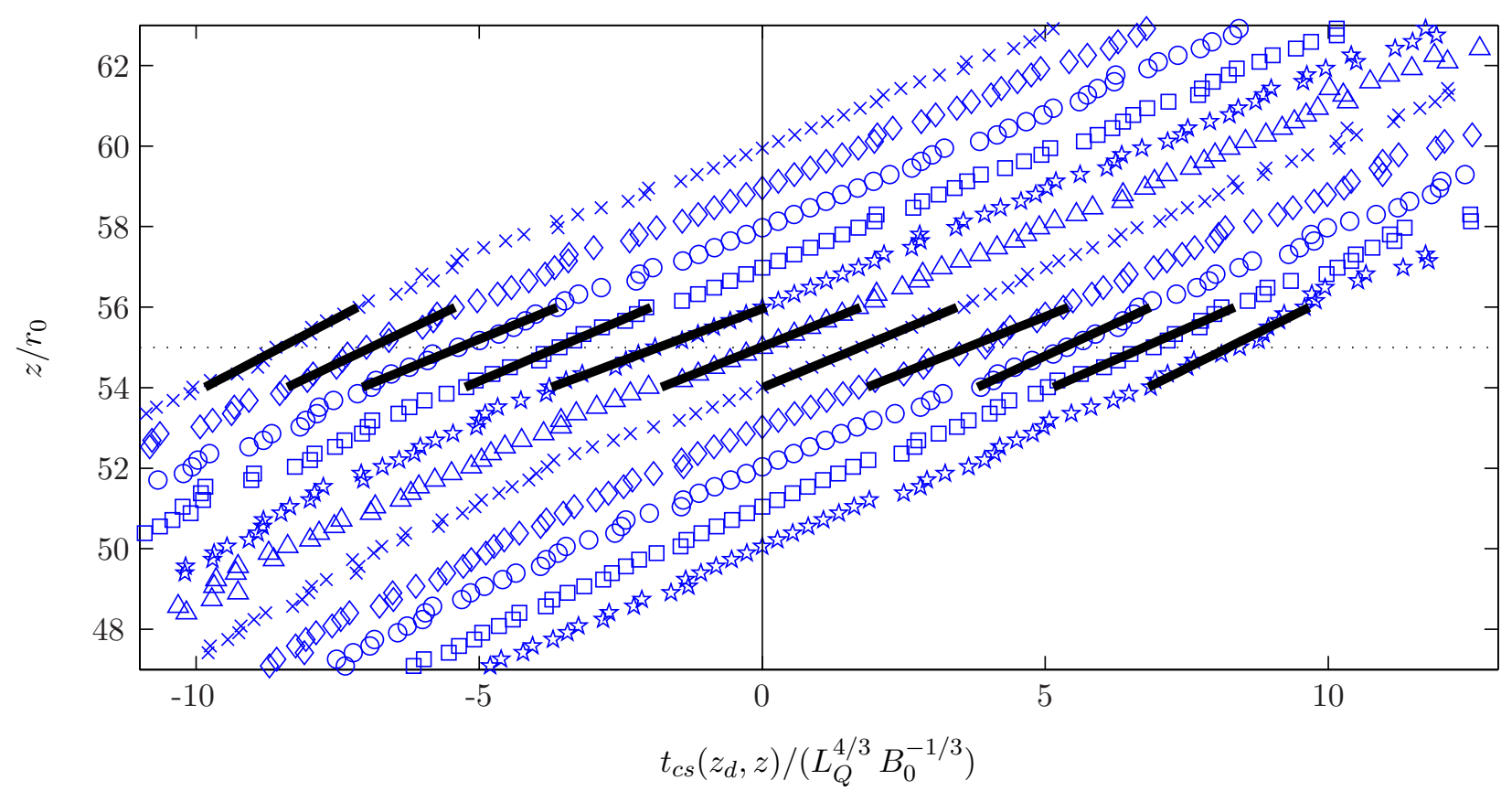

Fig. C1 The time taken, $t_{c s}\left(z_{d}, z\right)$, for coherent structures to travel from the height $z_{d}$ to the height $z$. The data from all of the eleven discrete heights $z_{d} / r_{0}=\{50,51,52,53,54,55,56,57,58,59,60\}$ (each marked by a particular symbol) from which velocity data at the height $z=55 r_{0}$ was inferred. The gradient of the thick black lines show the velocity of coherent structures at the height $z=55 r_{0}$, the average of which would provide our estimate of $w_{c s}\left(z=55 r_{0}\right)$ for the experiment shown.

which this minimum occurs then determines the threshold value for that pixel row. The plume edges for that row are defined as the first and last time that the light intensity falls below the threshold value. The location of the two edges was then stored and the algorithm is repeated for subsequent rows and then rows within subsequent images.

Despite our best efforts, the nature of the turbulent billows forming, advecting, merging and sometimes collapsing at the plume edge, on occasion, gives rise to uncertainty as to precisely where the plume edge is located. As such, for regions where the two algorithms returned significantly different results, data for that portion of the images (typically a region furthest from the source) was neglected for the particular experiment. For an example see figure 1b). 


\section{Appendix C. Determining velocities from time series of plume width}

From our measurements of plume width, with careful analysis and suitable ensemble averaging, we were able to reliably detect the local vertical velocities of coherent structures at the plume edge, $w_{c s}$. In order to do so, we cross-correlated the time series of plume widths at two different heights, both within the portion of the images at which the plume edges had been reliably determined. The time lag which maximised the correlation provided an indication of the time taken for coherent structures at the plume edge to travel between the two heights. We crosscorrelated the time series from various discrete heights $z_{d}$ with the time series from all other heights $z$ (pixel rows) at which we had data. In so doing for each experiment and for each $z_{d}$ we produced estimates of the time lag for the coherent structures to reach all heights $z$.

For any given experiment, the cross-correlations could be evaluated from time series constituting a subset of the recorded data. It was not obvious how to estimate the optimal length of time series, a priori. Indeed, the time at which we started recording was also arbitrary since the experiment was designed to be quasi-steady and so any given experiment could be continued indefinitely. We also desired to ensemble average estimates of the time lags in order to minimise uncertainty. As such for each pair of time series, we calculated cross-correlations taking a number of different time series, each a subset of the total recorded data at the two heights. We determined cross-correlations with the time series formed by taking with eleven different starting points within the recorded data (varied from the actual commencement of our recording up to time series starting $60 \%$ of the way through the recording, evenly spaced with increments of $5 \%$ ). For each of the eleven starting points we took cross-correlations with time series of six varying length portions of the remaining recorded data, varying in length from $50 \%$ to $100 \%$ of the remaining recorded data. For each pair of time series, taken at the heights $z_{d}$ and $z$, we therefore obtained 66 estimates of the time lag, averaging these 66 observations provided our estimate $t_{c s}\left(z_{d}, z\right)$ of the time taken for the coherent structure to travel from the height $z_{d}$ to $z$; note that $t_{c s}\left(z_{d}, z\right)<0$ is expected for $z<z_{d}$ and $t_{c s}\left(z_{d}, z\right)>0$ is expected for $z>z_{d}$. 
To reduce the computational time, we took the discrete heights $z_{d}$ to be vertically separated by distances approximately equal to one source radius $r_{0}$ (this corresponded to a separation of between 5 and 10 pixel rows in our experiments) throughout the vertical regions for which we had reliable data for the plume width. Figure C1 plots data from a typical experiment and shows the vertical variation of $t_{c s}\left(z_{d}, z\right)$ for the eleven different values of $z_{d}$ in the range $50 \leq z_{d} / r_{0} \leq 60$. The tracking of the coherent structures as they approached and passed through a particular $z_{d}$ proved successful for moderate vertical separations between the heights $z_{d}$ and $z$, typically for separations up to approximately $10 r_{0}$ (or approximately 50 and 100 pixel rows). For greater vertical separations the cross-correlations were not sufficiently good to produce reliable data. This is no doubt a result of a combination of new coherent structures (turbulent eddies) forming, merging or collapsing - increasingly likely to occur as the separation of $z_{d}$ and $z$ increased.

At a given height $z$, we calculated estimates for the velocity from the local vertical gradients in our measurements of the time $t_{c s}\left(z_{d}, z\right)$. Furthermore, we had sufficient information to calculate an independent estimate of the velocity, at any given $z$, based on cross-correlations with various different values of $z_{d}$, figure C1. For each height $z$ we calculated our estimate of the velocity of the coherent structures $w_{c s}$ by averaging eleven velocities inferred from the gradients in $t_{c s}\left(z_{d}, z\right)$ from cross-correlations at different heights, i.e. from the changes in $t_{c s}\left(z_{d}, z\right)$ for small changes in $z$ taking the eleven different measurements by varying $z_{d}$ in the range $z-5 r_{0} \leq z_{d} \leq z+5 r_{0}$. Figure $\mathrm{C} 1$ provides an illustration of gradients which were averaged to produce our estimate of the velocity $w_{c s}$ at $z=55 r_{0}$.

\section{References}

Burridge, H. C. \& Hunt, G. R. 2013 The rhythm of fountains: the length and time scales of rise height fluctuations at low and high Froude numbers. J. Fluid Mech. 728, 91-119.

Burridge, H. C. \& Hunt, G. R. 2016 The dynamical and physical behaviour of forced 
fountains inferred from coherent structures in the counterflow. J. Fluid Mech. In preparation.

CAnny, J. 1986 A computational approach to edge detection. IEEE Transactions on Pattern Analysis and Machine Intelligence (6), 679-698.

Craske, J. \& van Reeuwijk, M. Under consideration Generalised unsteady plume theory. J. Fluid Mech..

Crone, T. J., McDuff, R. E. \& Wilcock, W. S. D. 2008 Optical plume velocimetry: A new flow measurement technique for use in seafloor hydrothermal systems. Experiments in fluids 45 (5), 899-915.

Crone, T. J. \& Tolstoy, M. 2010 Magnitude of the 2010 gulf of mexico oil leak. Science 330 (6004), 634-634.

Ezzamel, A., Salizzoni, P. \& Hunt, G. R. 2015 Dynamical variability of axisymmetric buoyant plumes. J. Fluid Mech. 765, 576-611.

Haller, G. 2015 Lagrangian coherent structures. Ann. Rev. Fluid Mech. 47, 137-162.

Hussain, A. K. M. F. 1983 Coherent structures - reality and myth. Phys. Fluids 26 (10), $2816-2850$.

Hussain, A. K. M. F. \& Clark, A. R. 1981 On the coherent structure of the axisymmetric mixing layer: a flow-visualization study. J. Fluid Mech. 104, 263-294.

Landel, J. R., Caulfield, C. P. \& Woods, A. W. 2012 Meandering due to large eddies and the statistically self-similar dynamics of quasi-two-dimensional jets. J. Fluid Mech.692, $347-368$.

Mcnutt, M. K., Camilli, R., Crone, T. J., Guthrie, G. D., Hsieh, P. A., Ryerson, T. B., Savas, O. \& Shaffer, F. 2012 Review of flow rate estimates of the deepwater horizon oil spill. Proc. Natl. Acad. Sci. U.S.A. 109 (50), 20260-20267.

Morton, B. R. 1959 Forced plumes. J. Fluid Mech. 5, 151-163.

Morton, B. R., Taylor, G. \& Turner, J. S. 1956 Turbulent gravitational convection from maintained and instantaneous sources. Proc. R. Soc. Lond. A 234, 1-23.

Papantoniou, D. \& List, E. J. 1989 Large-scale structure in the far field of buoyant jets. 
J. Fluid Mech. 209, 151-190.

van Reeuwijk, M. \& Craske, J. 2015 Energy-consistent entrainment relations for jets and plumes. J. Fluid Mech. 782, 333-355.

Rocco, S. \& Woods, A. W. 2015 Dispersion in two-dimensional turbulent buoyant plumes. J. Fluid Mech. $\mathbf{7 7 4 .}$

Shabbir, A. \& George, W. K. 1994 Experiments in a round turbulent buoyant plume. J. Fluid Mech. 275, 1-32.

WANG, H. \& LAW, A. W-K. 2002 Second-order integral model for a round turbulent buoyant jet. J. Fluid Mech. 459, 397-428.

Woodhouse, M. J., Hogg, A. J., Phillips, J. C. \& Sparks, R. S. J. 2013 Interaction between volcanic plumes and wind during the 2010 Eyjafjallajökull eruption, iceland. J. Geophys. Res. Solid Earth 118 (1), 92-109.

Woods, A. W. 2010 Turbulent plumes in nature. Ann. Rev. Fluid Mech. 42, 391-412. 